Please do not remove this page

RMIT

UNIVERSITY

\title{
A low-power reconfigurable logic array based on double-gate transistors
}

Beckett, Paul

https://researchrepository.rmit.edu.au/esploro/outputs/9921863554801341/filesAndLinks?institution=61RMIT_INST\&index=null

Beckett, P. (2008). A low-power reconfigurable logic array based on double-gate transistors. IEEE Transactions on Very Large Scale Integration Systems, 16(2), 115-123.

https://doi.org/10.1109/TVLSI.2007.912024

Published Version: https://doi.org/10.1109/TVLSI.2007.912024

Repository homepage: https://researchrepository.rmit.edu.au

(c) 2007 IEEE. Personal use of this material is permitted. However, permission to reprint/republish this material for advertising or promotional purposes or for creating new collective works for resale or redistribution to servers or lists, or to reuse any copyrighted component of this work in other works must be obtained from the IEEE.

Downloaded On 2023/04/26 21:47:01 +1000 


\title{
A Low-Power Reconfigurable Logic Array Based on Double-Gate Transistors
}

\author{
Paul Beckett, Member, IEEE
}

\begin{abstract}
A fine-grained reconfigurable architecture based on double gate technology is proposed and analyzed. The logic function operating on the first gate of a double-gate (DG) transistor is reconfigured by altering the charge on its second gate. Each cell in the array can act as logic or interconnect, or both, contrasting with current field-programmable gate array structures in which logic and interconnect are built and configured separately. Simulation results are presented for a fully depleted SOI DG-MOSFET implementation and contrasted with two other proposals from the literature based on directed self-assembly.
\end{abstract}

Index Terms-CMOS integrated circuits, double-gate (DG) transistors, logic circuits, nanotechnology, reconfigurable architectures.

\section{INTRODUCTION}

$\mathbf{R}$ ECONFIGURABLE architectures are of great interest to system designers because they offer a way of achieving power and performance efficiency by matching specific algorithmic constructs with an appropriate architecture [1]. The traditional approach to developing reconfigurable systems, in fieldprogrammable gate arrays (FPGAs), for example, has been to build separate regions of programmable logic gates and interconnection and to manage these two resources more-or-less separately during the synthesis process. Therefore, much of the work on reconfigurable platforms has been directed towards answering questions such as "how much of each and in what form?" (e.g., [2]).

The reduced fan-out, power handling capacity, gain, and reliability of deep-submicrometer (DSM) and nanoscale devices will have a number of consequences for reconfigurable systems. As device dimensions shrink, it will become increasingly difficult to manufacture the complex heterogeneous layouts that have underpinned field-programmable technology to date. Physical issues such as the increasing difficulty in achieving alignment between process layers [3] as well as the prospect of poor performance of FETs at reduced gate lengths [4] have already forced designers to look towards alternative manufacturing techniques on which to base programmable architectures. Ideas such as chemically-assembled molecular electronics [5], nanotube and nanowire devices [6], quantum dot techniques [7], and magnetic spin-tunneling devices [8] have all been proposed as the basis of future reconfigurable systems.

This research is motivated by two related questions: what types of simple (regular) CMOS structures can be exploited to create future reconfigurable architectures at nanoscale dimensions and how might heterogeneous functionality emerge

Manuscript received May 10, 2006; revised March 19, 2007.

The author is with the School of Electrical and Computer Engineering, RMIT

University, Melbourne 3000, Australia (e-mail: pbeckett@rmit.edu.au).

Digital Object Identifier 10.1109/TVLSI.2007.912024 from an essentially homogeneous array of simple devices? Remaining in the CMOS domain offers a number of advantages, including the availability of three terminal switching devices with intrinsic gain, a stable and well characterized manufacturing base plus compatibility with existing design tools. The disadvantage is that the design is constrained by lithographic patterning and alignment issues. While it is forecast that feature sizes (for logic) will reduce below $20 \mathrm{~nm}$ by 2016-2018 [9], it is not clear at the moment how this might be achieved. Our premise here is that simplified, regular structures with a minimal number of interconnection layers will have a better chance of achieving sub-20 nm feature sizes than the complex, heterogeneous layouts that characterize most current micro-architectures. It is likely that a "wish-list" of features for future reconfigurable architectures would include at least some of the following:

- a simplified processing technology;

- a highly regular layout style;

- small logic and interconnect footprints, supporting high component densities;

- configuration flexibility supporting efficient routing as well as allowing a continuous tradeoff between routing and logic;

- an organization that minimizes reconfiguration overheads.

The double-gate (DG) transistor is a promising device applicable to DSM due in particular to its inherent resistance to short-channel effects and potentially ideal subthreshold performance. Typically, the two gates would be operated together as this offers the best switching performance. However, accessing the two gates separately creates opportunities for innovative circuit design [10]. This paper proposes a reconfigurable architecture based on double gate transistor circuits where the operating point of the circuit can be set via one gate while the other gate is used to form the logic array. In this way, the overheads imposed by reconfigurability can be reduced or hidden to an extent where it becomes possible support complex datapath architectures with homogeneous fine-grained organization.

The remainder of this paper proceeds as follows. In Section II, the operation of DG device is introduced and its application to a reconfigurable logic cell is described. Section III then shows how these cells can be assembled to form a homogenous reconfigurable processing mesh capable of flexible configuration into logic and/or interconnect. We also present some preliminary performance estimates. Finally, this paper is summarized in Section IV.

\section{RECONFIGURABLE DG CELL}

The many problems associated with scaling MOS transistors are likely to result in the DG transistor becoming a preferred circuit element. They are predicted by the ITRS [9] to appear as early as 2011. Theoretically, these devices do not need channel doping and, therefore, can be scaled to dimensions below 10 


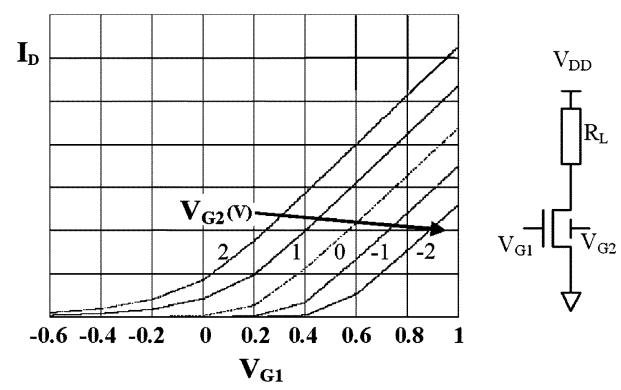

Fig. 1. Generalized DG transistor characteristics, showing $\mathrm{I}_{D}-V_{G 1}$ over a range of $V_{G 2}$.

$\mathrm{nm}$ without running into problems of uncontrollable parameter variations due to the random distribution of dopant atoms [11]. This section outlines the basic operation of the DG transistor and shows how it supports the proposed reconfigurable logic array. We first look at the generic behavior of these devices and then at a specific implementation using silicide source/drain that may result in simplified processing and superior performance as an "end-of-roadmap" device.

\section{A. DG Transistor Operation}

It appears likely that in typical applications DG devices will be set up to use the front and back gates together as this leads to the best performance as a switching device [12]. However, if the two gates can be accessed independently, one can be used to set the operating point of the transistor thus affecting the threshold behavior of the other. This is the basis for the operation of the proposed cell. Fig. 1 shows the basic idea for a generic double-gate $n$-channel device simulated in Spice-3 using a fully depleted SOI model developed at the University of Florida [13]. For this example, the transistor models were approximately calibrated to the idealized SOI double-gate device described in [14]. The impact of shifting the gate-source bias on one gate $\left(V_{G 2}\right.$ in this case) is to move the effective threshold voltage $\left(V_{\mathrm{TH}}\right)$ seen at the other. Similar behavior occurs in the p-channel devices. The shift in threshold is accompanied by a change in the subthreshold slope, which will be discussed later in this section. It should be noted that this effect is entirely symmetrical, and of equal magnitude if both gate oxide thicknesses are the same. In the following, we will tend to refer to one gate as the control gate and the other as the logic gate.

As most of the important operating parameters of a logic gate (e.g., switching threshold, gain, noise margin, etc.) depend on $V_{\mathrm{TH}}, V_{G 2}$ may be used to vary these during operation. The shift in the logic switching threshold is illustrated in Fig. 2 for a simple inverter circuit under five operating conditions $\left(V_{G 2}=\right.$ $1.5,0.5,0,-0.5$, and $-1.5, V_{\mathrm{DD}}=1 \mathrm{~V}$, no load). At the two extremes, the output remains high $\left(V_{o}>0.8 \mathrm{~V}\right)$ or low $\left(V_{o}<\right.$ $0.1 \mathrm{~V}$ ) for all input values whereas for $V_{G 2} \approx 0 \mathrm{~V}$, the output switches symmetrically. The resulting transfer curves are similar to the characteristics of the planar "ground plane" (GP) CMOS inverter presented by Ieong et al. in [15]. One objective of that investigation was how to use the bias on the second gate to improve noise margins and gain for the inverter circuit so those experiments focused only on part of the bias range $\left(V_{G 2}<0\right)$. In the case of the reconfigurable cell proposed in

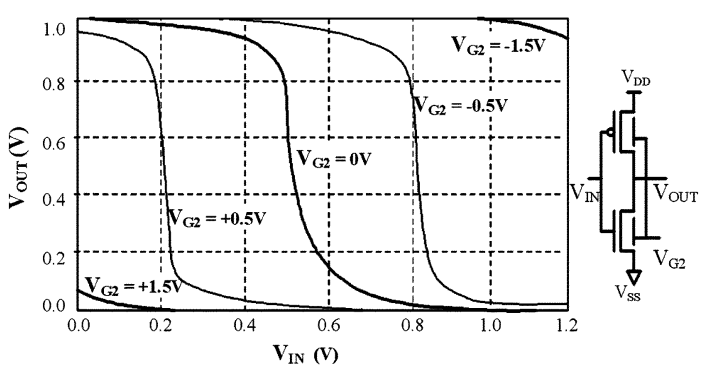

Fig. 2. Switching characteristics of a configurable inverter over a range of control voltages $V_{G 2}$.

this paper, the offset is used at least partly in the opposite direction: to reduce the gain to as close to zero as possible.

Several DG topologies have been described in both MOS and heterojunction technologies (e.g., [16]-[18]). Of these, the back-gate planar configuration [18] may result in the densest cell layout for this particular application. Although achieving close alignment between the gates still represents a major processing hurdle, process flows for planar DG-MOSFET with selfaligned top and bottom gates have already been demonstrated [12], [18], and there is evidence to suggest that adequate performance might be achieved without precise gate alignment [19]. Although these devices have focussed on conventionally doped source/drain devices, an alternative using silicide source/drain regions and a mid-band metal gate structure may offer some advantages and this is briefly explored in Section II-B.

\section{B. Silicide Source/Drain Devices}

As previously mentioned, performance fluctuations due to random dopant distribution in the channel will become a major problem in CMOS at reduced gate lengths. An alternative approach, employing an undoped channel region and using Schottky barriers at the source and drain has been demonstrated by a number of researchers. Metal silicides form natural Schottky barriers to silicon substrates, acting to confine carriers and reducing or eliminating the need for impurities in the channel to prevent current flow in the off condition [20]. Schottky barrier devices were first described more than 30 years ago [21] and have been investigated for many years. Although they generally exhibit significantly lower drive currents than conventional devices, this gap may close as devices shrink. For example, there is evidence that quantum confinement effects in thin, narrow silicon wires (i.e., nanowires) may result in quasi-ballistic operation leading to greater mobility values than in bulk silicon [14], although increased scattering due to edge roughness and other effects may ultimately prevent fully ballistic behavior [23].

As part of this paper, we have simulated a number of DG thin-body Schottky devices of the form shown in Fig. 3 using a commercial simulator with classical transport models [24]. The results were then compared with the characteristics of previously reported devices (in [25] and [26], for example). All of the physically implemented devices reported to date have been either single-gate or planar. Thus, the objective of this simulation work was to determine the likely extent of the threshold shift in intrinsic channel, DG, silicide S/D SOI devices.

The source/drain regions of the n-type transistors were assumed to use $\mathrm{ErSi}_{1.7}$ (barrier height, $\psi_{b n}=0.28 \mathrm{eV}$ above $\mathrm{Si}$ ) although in [27] it is shown that Ytterbuim Silicide $\left(\mathrm{YbSi}_{2-x}\right)$ on silicon exhibits an electron barrier of $\sim 0.27 \mathrm{eV}$ and may be 


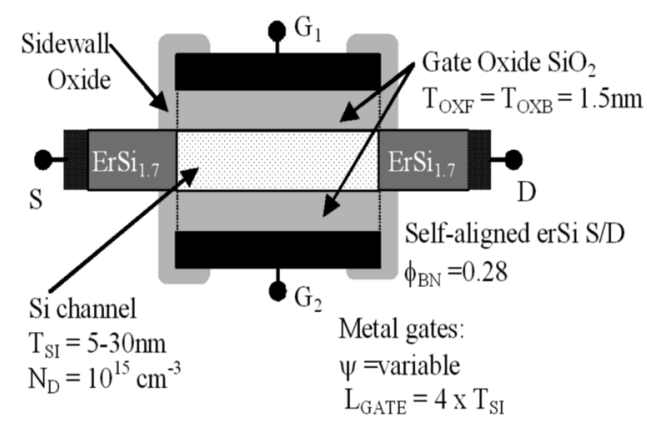

Fig. 3. Basic topology of the thin-body DG transistor with silicide source/drain.

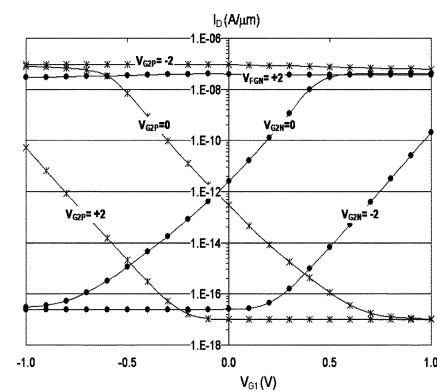

Fig. 4. Simulated $I_{D}$ versus $V_{B 1}$ characteristics of the transistors of Fig. 3-n-type (dots), p-type (crosses) with $\mathrm{p}$ and $\mathrm{n}$ control gate biasses $V_{G 2 P}$ and $V_{G 2 N}=-2,0,+2$ as labeled.

easier to fabricate in thin films than $\mathrm{ErSi}_{1.7}$. PtSi was assumed for the p-type devices $\left(\psi_{b p}=0.23 \mathrm{eV}\right)$. In both cases the gate material was assumed to be $\mathrm{Au} / \mathrm{Cr}$ with a work function of about $4.7 \mathrm{eV}$. The width and length were both fixed at $20 \mathrm{~nm}$ and the gate oxide thickness at $1.5 \mathrm{~nm}$.

Although we used classical diffusion models without quantum correction, these have been shown to be sufficiently accurate to the limit of this work around $T_{\mathrm{SI}}=5 \mathrm{~nm}$ [28]. However, the models appeared to underestimate the drain current density at these dimensions. For example, the value of $I_{\mathrm{ON}} \approx 0.1 \mu \mathrm{A}$ derived here contrasts with actual devices measured in [25] which exhibited current drives in the order of $2 \mu \mathrm{A}$ for the 20 -nm thick channel in their study. We, therefore, consider our drive current results to be a worse-case prediction. In addition, it has been shown that quantum confinement effects can produce a large threshold voltage shift in DG devices below $30 \mathrm{~nm}$ gate lengths [29], an effect that is not accounted for here. Neither of these effects was important to this part of the study and in any case, the quantum $V_{\mathrm{TH}}$ shift is most pronounced below $\mathrm{L}_{G}=10 \mathrm{~nm}$.

Fig. 4 shows the simulated $I_{D}$ versus $V_{G 1}$ performance for these devices with the control gate voltages $\left(V_{G 2}\right)$ set between $\pm 2 \mathrm{~V}$, indicating that values of $I_{\mathrm{ON}} / I_{\mathrm{OFF}}>10^{4}$ can be achieved using these devices and that a significant threshold shift can be expected as the control gate is modulated (e.g., $\left.\Delta V_{\mathrm{TH}} / \Delta V_{G 2}=0.375 \mathrm{~V}\right)$. This is the basis of the operation of the proposed devices that can be exploited to create reconfigurable lookup table (LUT) structures from simple arrays, as will be discussed in Section II-C.

Fig. 5 illustrates the relative sensitivity of the threshold voltage seen at the logic gate to the control gate bias with various values of channel thickness $\left(T_{\mathrm{SI}}\right)$ between 5 and $30 \mathrm{~nm}$. These plots are for the n-type transistor; those for the p-type

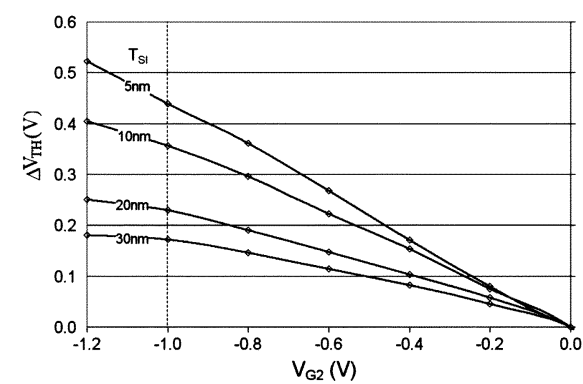

Fig. 5. Threshold voltage change $\left(\delta V_{\mathrm{TH}}\right)$ versus control gate voltage $\left(V_{G 2}\right)$ at various $T_{\mathrm{SI}}$ for the n-type device of Fig. 3 . The shape and magnitude of the P-type device characteristics are similar.

have a similar form and magnitude. Here the threshold values have been normalized such that $V_{\mathrm{TH}}=0$ at $V_{\mathrm{BG}}=0$. As the channel thickness is reduced the threshold sensitivity increases to a point where at $T_{\mathrm{SI}}=5 \mathrm{~nm}$, setting $V_{\mathrm{BG}}=-1 \mathrm{~V}$ can produce $0.45-\mathrm{V}$ shift in threshold voltage with a shift of similar magnitude observed for the p-type device at $V_{\mathrm{BG}}=V_{\mathrm{DD}}+1$.

In the ground-plane mode (i.e., with one gate at a fixed potential), the behavior of the subthreshold slope (S) is similar to that of planar devices and is given by [30]

$$
S=\frac{k T}{q} \ln (10)\left[1+\frac{C_{S}}{C_{\mathrm{OX} 1}}\right]
$$

where $C_{\mathrm{OX} 1}=$ oxide capacitance of gate $1 \approx \varepsilon_{\mathrm{OX}} / T_{\mathrm{OX} 1}$ and $C_{S}$ is the effective body capacitance between the inversion layer and gate 2 (see Fig. 3 ) so that $C_{S} \approx \varepsilon_{\mathrm{SI}} / T_{\mathrm{SI}}$ if the back surface is in accumulation, and $C_{S}=C_{\mathrm{SI}} C_{\mathrm{OX} 2} /\left(C_{\mathrm{SI}}+C_{\mathrm{OX} 2}\right)$ in depletion. Substituting $\varepsilon_{\mathrm{SI}} \approx \kappa_{r} \varepsilon_{\mathrm{OX}},\left(\kappa_{r}=\varepsilon_{\mathrm{SI}} / \varepsilon_{\mathrm{OX}} \approx 3\right.$ for $\mathrm{SiO}_{2}$ dielectric), the subthreshold slope becomes

$$
S \approx 60\left[1+\frac{\kappa_{r} T_{\mathrm{OX} 1}}{\kappa_{r} T_{\mathrm{OX} 2}+T_{\mathrm{SI}}}\right] \mathrm{mV} / \text { decade. }
$$

The term $\kappa_{r} T_{\mathrm{OX} 2}$ becomes zero when that surface is in accumulation. This implies that scaling the silicon body thickness to $5 \mathrm{~nm}$ will require the oxide thickness to shrink to less than $1 \mathrm{~nm}$ to maintain $S<100 \mathrm{mV} /$ decade (which, in turn, will restrict the supply range for reasons related to gate tunneling and oxide breakdown). While is theoretically possible for DG-SOI transistors to approach the ideal subthreshold slope for MOS ( $\sim 60 \mathrm{mv} /$ decade) when used in DG mode (both gates driven together), none of the nMOS devices reported to date has reached this figure, although the $\mathrm{YbSi}_{2-x} \mathrm{~S} / \mathrm{D}$ bulk device described in [27] achieves $S=75 \mathrm{mV} /$ decade. Previous values (e.g., [31]) have ranged between 100 and $150 \mathrm{mV} /$ decade, i.e., $\mathrm{C}_{S} / \mathrm{C}_{\mathrm{OX}}$ between 0.6 and 1.5 .

While the threshold sensitivity $\left(\Delta V_{\mathrm{TH}} / \Delta V_{\mathrm{BG}}\right)$ increases with reducing the body thickness, the subthreshold slope degrades. It is suggested in [28] that this limits the $V_{\mathrm{TH}}$ tuning range (to $V_{\mathrm{BG}}<0.25$ in that study). However, in our application the subthreshold shift is still useful well outside this range, regardless of the final value of S. It can also be seen from (4) that moving to hi- $\kappa$ gate dielectrics can significantly improve $\mathrm{S}$ in this case (as well as serving to reduce gate leakage). For example, using $\mathrm{HfSiO}_{4}\left(\varepsilon_{\mathrm{OX}}>12\right), \kappa_{r}$ in (2) becomes less than 1.0 and the worse-case slope will reduce to approximately $78 \mathrm{mV} /$ decade, although it is likely that this will be at the expense of reduced channel mobility and increased short channel effects [32]. 


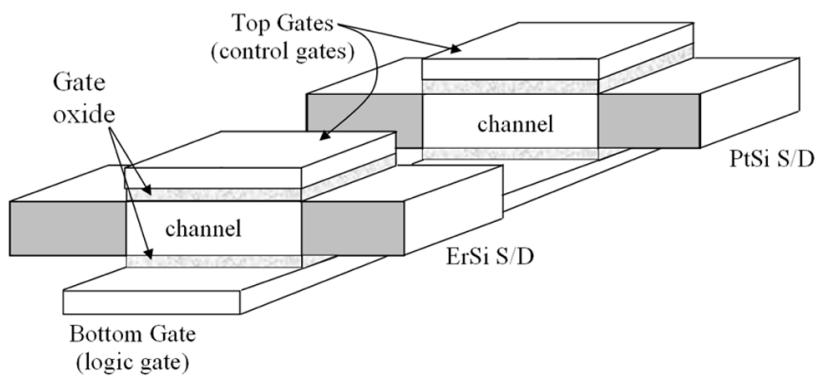

Fig. 6. Simplified view of the Schottky source/drain transistor pair.

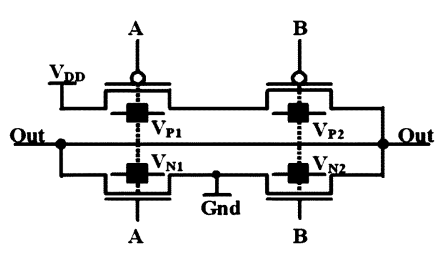

\begin{tabular}{|c|c|c|c|c|}
\cline { 2 - 5 } \multicolumn{1}{c|}{} & \multicolumn{4}{c|}{ Control gate voltages $(\mathrm{V})$} \\
\hline $\boldsymbol{f}(\mathbf{A}, \mathbf{B})$ & $\mathbf{V}_{\mathbf{P} 1}$ & $\mathbf{V}_{\mathbf{N} \mathbf{1}}$ & $\mathbf{V}_{\mathbf{P} \mathbf{2}}$ & $\mathbf{V}_{\mathbf{N} \mathbf{2}}$ \\
\hline$\overline{\mathrm{A}}$ & 1 & 0 & 0 & -1 \\
\hline$\overline{\mathrm{B}}$ & 0 & -1 & 1 & 0 \\
\hline$\overline{\mathrm{A}+\mathrm{B}}$ & 1 & 0 & 1 & 0 \\
\hline 1 & 0 & -1 & 0 & -1 \\
\hline 0 & 2 & 1 & 2 & 1 \\
\hline
\end{tabular}

(a)

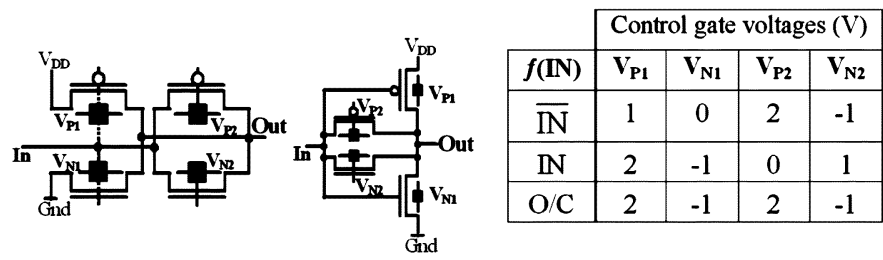

(b)

Fig. 7. Configurable cells based on DG transistors. (a) Configurable 2-NOR gate. (b) Configurable pass gate/inverting buffer structure.

\section{Assembling the Array}

Having now described the operation of its components, we can now show how the array can be assembled. The objective here is to achieve a simple, regular organization that can then be configured using the control-gates of the complementary transistors. One possible layout for such an array is based on pairs of $\mathrm{p}$ and $\mathrm{n}$-type transistors formed in undoped silicon by depositing (and annealing) materials such as erbium and platinum silicide in alternate rows (see Fig. 6). Conventional flash memory techniques would be applicable to set the top gate charge in a similar way to the SOI Flash cell described in [33]. Other more speculative configuration mechanisms have been suggested in [34] and [35].

In Fig. 7, these complementary transistors have been organized into simple circuits exploiting this basic mechanism. Fig. 7(a) represents a configurable two-input NOR gate in which the threshold on each transistor is controlled via its gate bias voltage (shown as black squares on the diagram) so that the circuit can develop an enhanced set of logic functions shown in the accompanying table. The four-transistor cell in Fig. 7(b) can be configured to behave as either an inverting buffer or as a non-inverting pass gate. It can also be put into a high impedance mode to isolate its input and output signal. As will be shown below, together these two cell types are sufficient to create a "sea-of-gates" style configurable layout.
To analyze the characteristics of the 2-NOR gate, we can start with the equation for saturation drain current in DSM technology developed in [36]

$$
I_{D}(\mathrm{sat}) \approx\left(\frac{W}{\sqrt{L}}\right)\left(\frac{f\left(R_{s}\right)}{T_{\mathrm{OX}}^{0.8}}\right)\left(V_{\mathrm{GS}}-V_{\mathrm{TH}}\right)^{\alpha}
$$

where $L$ is the physical gate length, $T_{\mathrm{OX}}$ the effective gate oxide thickness, including any depletion layer effects, and $f\left(R_{s}\right)$ is a function of the source/drain resistance for a particular technology. The exponent $\alpha$ describes the degree of velocity saturation. It is given a value of 1.25 for DSM in [36], and will tend towards 1 in short-channel, ballistic devices [37]. Assuming that the term $W f\left(R_{s}\right) / \sqrt{L} T_{\mathrm{OX}}^{0.8}$ is constant for a given technology and circuit design style, we can equate the saturation drain currents in the conventional way [38] to derive an approximate formula for the switching threshold of the NOR circuit

$$
V_{\mathrm{SW}}(\mathrm{NOR})=\frac{V_{\mathrm{THN}}+n^{-1} K_{r}^{1 / \alpha}\left(V_{\mathrm{DD}}+V_{\mathrm{THP}}\right)}{1+n^{-1} K_{r}^{1 / \alpha}}
$$

where $V_{\mathrm{THN}(P)}$ is the threshold voltage for the $N(P)$ devices, $n$ is the number of transistors in the stack [e.g., 2 in Fig. 7(a)] and $K_{r}=\left(K_{P}\right) /\left(K_{N}\right)$. Thus, $K_{r}$ will be a function of the transistor gain ratio-given by $(W / \sqrt{L})_{P} /(W / \sqrt{L})_{N}$ and the relative mobilities (replaced in (1) by the $f\left(R_{s}\right) / T_{\text {OX }}$ term). It can be noted here that the effective mobility of the Schottky barrier pMOS device is greater than that of the nMOS due to the lower barrier height of PtSi (0.23 V versus $0.28 \mathrm{~V}$ for $\mathrm{ErSi}_{1.7}$, see Fig. 3). From (4), with $V_{\mathrm{THN}}=-V_{\mathrm{THP}}$ the switching threshold $\left(V_{\mathrm{SW}}\right)$ becomes $\left(V_{\mathrm{DD}}\right) /(2)$ when $n^{-1} K_{r}^{1 / \alpha}=1$ and thus

$$
K_{r}=n^{\alpha}
$$

It can also be seen from (4) that $V_{\mathrm{SW}} \geq V_{\mathrm{DD}}$ when

$$
V_{\mathrm{THN}}+n^{-1} K_{r}^{1 / \alpha} V_{\mathrm{THP}} \geq V_{\mathrm{DD}}
$$

and $V_{\mathrm{SW}} \leq 0 \mathrm{~V}$ when

$$
V_{\mathrm{THP}}+n^{-1} K_{r}^{1 / \alpha} V_{\mathrm{THN}} \leq-V_{\mathrm{DD}}
$$

which, together, describe the operation of the configurable NOR in Fig. 7(a). Analogously to a conventional $\mathrm{E}^{2}$ ROM, changes at the control gate shifts $V_{\mathrm{THN}}$ and $V_{\mathrm{THP}}$ for a given input sufficiently that the switching threshold seen that input moves outside the voltage range of the logic signal. This is illustrated in Table I which lists $V_{\text {THN }}$ and $V_{\text {THP }}$ for one input of Fig. 7(a) as well as the overall switching threshold seen at that input derived from (4). "Normal operation" means that the switching threshold is about $V_{\mathrm{DD}} / 2$ and the gate is sensitive to that input. Setting large but symmetrical threshold values puts the array in a low standby power mode. While $V_{\mathrm{SW}}$ is still approximately $V_{\mathrm{DD}} / 2$, the propagation delay is severely affected so that the cell would not normally be operated in this mode. Shifting its switching threshold to greater than $V_{\mathrm{DD}}$ effectively makes the gate insensitive to that input. Similarly, when its threshold is less than ground, the n-device will be always conducting. As this is a NOR configuration, any transistor pair in this condition results in an output low.

Equations (4)-(7) also illustrate two further points about this organization. First, as the supply reduces with scaling, the range of threshold shifts that will be required to configure the array will also scale down. Second, although the optimum value of 
TABLE I

OPERATING CONDITIONS AT INPUT A OF THE CONFIGURABLE 2-NOR GATE OF Fig. 7(a), BASED ON THE Device ChaRACTERISTics IN Fig. $4\left(n=2, K_{r}=3, V_{\mathrm{DD}}=1 \mathrm{~V}\right)$

\begin{tabular}{|c|c|c|c|c|l|}
\hline $\begin{array}{c}V_{N 1} \\
(\mathrm{~V})\end{array}$ & $\begin{array}{c}V_{P 1} \\
(\mathrm{~V})\end{array}$ & $\begin{array}{c}V_{T H N} \\
(\mathrm{~V})\end{array}$ & $\begin{array}{c}V_{T H P} \\
(\mathrm{~V})\end{array}$ & $\begin{array}{c}V_{S W} \\
(\mathrm{~V})\end{array}$ & Comments \\
\hline 0 & +1 & 0.45 & -0.45 & 0.5 & Normal operation \\
\hline-1 & +2 & 0.82 & -0.82 & 0.47 & Low standby power \\
\hline-2 & 0 & 1.2 & -0.1 & 1.04 & logic line disabled \\
\hline+1 & +2 & -0.3 & -0.82 & -0.04 & output low \\
\hline
\end{tabular}

$K_{r}$ (i.e., such that $V_{\mathrm{SW}}=V_{\mathrm{DD}} / 2$ ) is related to $\alpha$, in common with all static gates we can adjust $K_{r}$ over a fairly wide range with a minimal effect on $V_{\mathrm{SW}}$ (but with an effect on performance [38]). For example, with $\alpha=1.25$ (at $V_{\mathrm{DD}}=0.8 \mathrm{~V}$, $V_{\mathrm{THN}}=\left|V_{\mathrm{THP}}\right|=0.3 \mathrm{~V}$ ), setting $K_{r}=4$ will result in $V_{\mathrm{SW}} \approx 0.37 \mathrm{~V}$ (i.e., $\sim 4 \%$ shift from its optimum value). We can also achieve a shift in switching between $V_{\mathrm{DD}}$ and $0 \mathrm{~V}$ with $\Delta V_{\mathrm{TH}} \approx \pm 0.45 \mathrm{~V}$, which would require the front gate to be modulated by approximately $\pm 1.3 \mathrm{~V}$. This is consistent with the results for the simulated device shown in Fig. 5 at $T_{\mathrm{SI}}=5 \mathrm{~nm}$.

Up to this point, we have ignored the impact of threshold voltage variability, which will increase the range of gate biases necessary to achieve the desired switching thresholds. For example, substituting into (4) a worse-case $V_{\mathrm{TH}}$ value with $+3 \sigma_{V_{\mathrm{TH}}}=+25 \%$ of the nominal $V_{\mathrm{TH}}$ for both the $\mathrm{p}$ and $\mathrm{n}$ devices (twice the ITRS figure of $\sim 12 \%$ ), and assuming the same $\Delta V_{\mathrm{TH}}$ as above $( \pm 0.45 \mathrm{~V})$, results in the maximum bias increasing from $\pm 1.3 \mathrm{~V}$ to approximately $\pm 2 \mathrm{~V}$. Thus, we expect that gate biases in the range of $\pm 2 \mathrm{~V}$ (that are also compatible with oxide reliability [39]) will be sufficient to configure this array.

We will demonstrate the operation of this reconfigurable cell using the organization shown in Fig. 8 which is set up as a 6-input, 6-output NOR-based LUT with each output line terminated in a configurable inverter/3-state driver as described in Fig. 7(b). Alternative organizations (e.g., NAND-LUT) would be equally possible with a slight rearrangement of the internal connections. These cells are then organized with adjacent connections in the vertical and horizontal directions plus two local feedback connections (see Fig. 9), making an $8 \times 8$ reconfiguration block at each cell position. Arranged in this way, each pair of adjacent cells contains sufficient resources for either a small combinational logic circuit such as a 3-LUT, more complex synchronous state machine elements such as latches and flip-flops or simple asynchronous circuits, as will be described in Section III.

\section{Reconfigurable Computing Circuits}

The reconfigurable array described in Section II could be said to be "polymorphic" [34], [35] in that it may be arbitrarily configured as logic and/or interconnect or as combinations of both. Although there are many ways to implement such an array, the thin-body, fully-depleted, DG MOSFET devices described in Section II at least have the advantage that they represent a plausible evolutionary path from conventional CMOS technology. In this section, we demonstrate that the simple array structure proposed in Section II can support a range of complex datapath architectures.

\section{A. Programmable Interconnect}

A primary characteristic of this proposed array is that there is little intrinsic difference between logic and routing and each

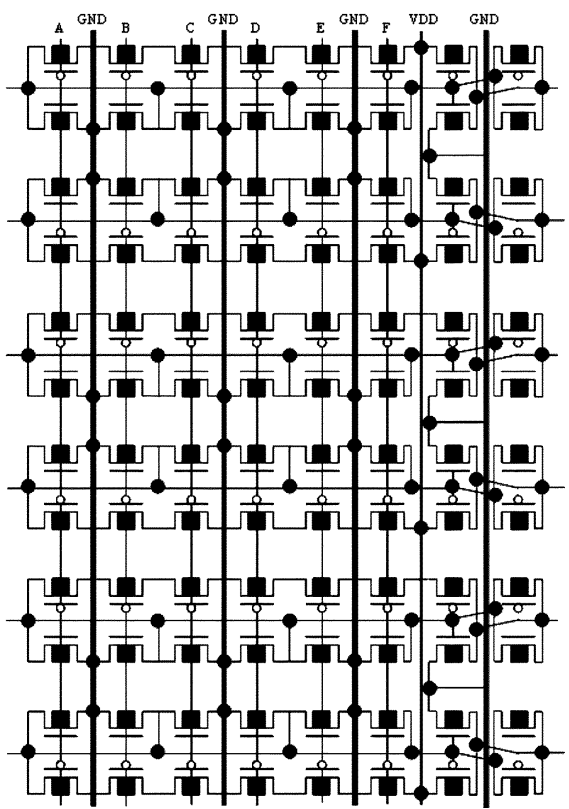

Fig. 8. Single reconfigurable cell based on a $6 \times 6$ NOR organization with pass/ invert interface gates. The black squares are the top gate programming nodes.

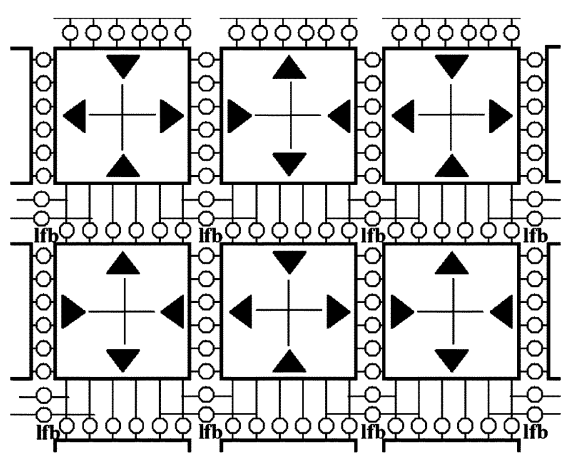

Fig. 9. Data flow in adjacent cells. The open circles are the pass/invert interface cells between 6-LUTs. The corner space between groups of four cells is occupied by additional sets of pass/invert cells for local interconnect.

cell can be used for both simultaneously. Fig. 10 illustrates the range of options available to merge logic fan-in, fan-out, and routing as follows.

1) Logic Fan-in and Fan-out: The basic layout of each cell comprises of six programmable 6-NOR gates [see Fig. 7(a)]. Input lines that are not part of a particular logic mapping on a 6-NOR are set to "don't care" on that gate by shifting the effective switching threshold seen at that input. In the example of Fig. 10, the nine inputs $A, \ldots, I$ are partitioned across cells 1,3 , and 5 . Cell 1 creates the logic function $G+H+I$ (normal threshold for these three inputs, high $\left|V_{\mathrm{TH}}\right|$ for the remaining three). The term is then routed across cell 2 and 3 to merge with the terms $A+B+C$ and $D+E+F$ generated by cells 3 and 5 , respectively. The resulting function appears on line "Y" at the output of cell 4 and is then transferred to cell 9 . In a similar manner, this term is distributed horizontally to cells 8 and 11 where it is combined with inputs routed through 2, 6, 7, and 11 . The partial logic terms developed in cells 8 and 10 are then transferred via 12 and 14 to be recombined in cell 13. (Note that this example is not intended to represent any particular logic function). 


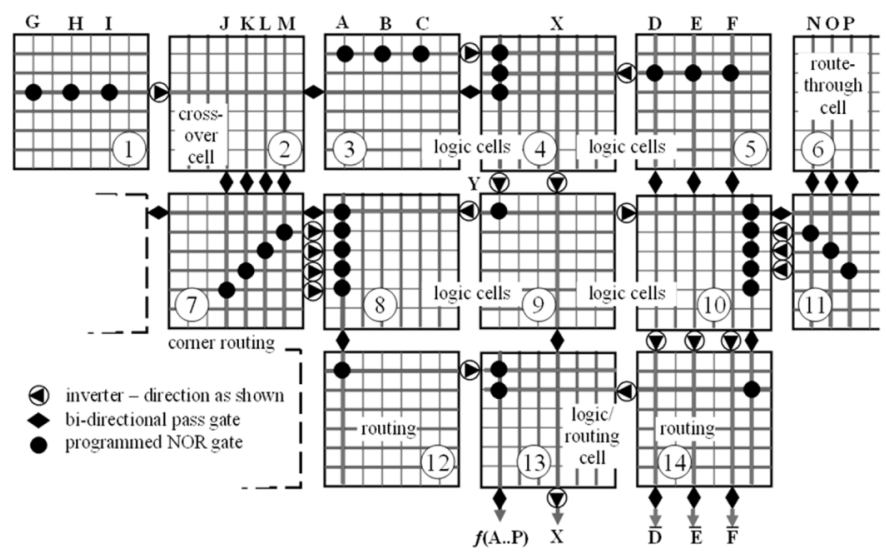

Fig. 10. Programmable cell styles: each cell can be set up as configurable NOR logic and/or routing block. Linking two adjacent cells via a (bidirectional) pass gate allows signals to be routed across a cell to the next logic gate.

2) Routing: With a few restrictions, signal routing can occur simultaneously in the two orthogonal directions within each cell. First, any input line automatically carries that signal across the cell so that it is available to be connected to an adjacent cell via a pass transistor. The partial directionality imposed by the interface gates restricts this option to noninverting (pass) logic. At the same time, any output line not involved in logic, for which all transistors are biased "off" (i.e., high $\left|V_{\mathrm{TH}}\right|$ ) can also be used to route signals across the cell. As an example, cell 4, which is used to form the intermediate logic terms previously outlined, is also shown transferring the signal " $X$ " vertically to cell 9 , from where it continues through to cell 13. Finally, each cell is effectively equivalent to a $6 \times 6$ universal routing block (e.g., cells 7 and 11 in Fig. 10). These corner routing cells can be inverting or noninverting and can be easily cascaded. The result is a flexible organization that supports fine-grained tradeoffs between logic and routing. Ironically, this level of flexibility is also likely to make the job of automatic place-and-route difficult and as a result it might not be possible to maximize the use of every individual cell.

\section{B. Combinational and Sequential Logic}

As an example of combinational and sequential logic synthesis, a simple D-type flip-flop and a full-adder circuit were simulated using Spice3 level 10 SOI models available at the Nanotechnology Simulation Hub [40]. The thin-film double gate transistor models of the D-type were tuned approximately to the characteristics of the devices fabricated in [25] (with a particular focus on the subthreshold slope) while the full-adder used parameters derived from the simulated devices outlined in Section II. Figs. 11 and 12 show the results for the D-type flip-flop and full-adder, respectively. Table IV summarizes the performance of these circuits and, as a comparison, also includes simulation results from [41] and [42] that are further discussed in Section III-D.

\section{Layout Area Comparisons}

In this section, we estimate the area of circuits on our array and compare it in general terms with the fixed channel islandstyle routing of a conventional FPGA. The primary objective here was to explore whether complex logic can be mapped efficiently onto a flat, undifferentiated array of the sort proposed in

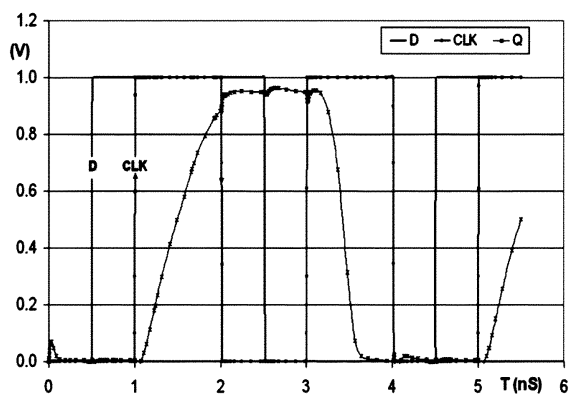

Fig. 11. Simulated waveforms for D-type FF circuit $\left(V_{\mathrm{DD}}=1.0 \mathrm{~V}\right)$.

this paper and whether this fine-grained array style can support larger datapath components.

We start with an analysis of the netlists of a number of circuits drawn from the LGSynth93 archive [43]. Each of these circuits had already been partitioned onto a basic logic element (BLE) comprising a 4-LUT and a flip flop [see Fig. 13(a)]. The component values listed in Table II represent the number of cells mapped a particular basic block configuration, i.e., LUT only (bypassing the D flip-flop), latch only (bypassing the LUT) and both LUT and flip-flop simultaneously. Table II also shows the estimated area required by these circuits. We did not actually place and route these circuits, but based the estimates on a number of the following conservative assumptions.

1) In the PMA case, a 4-LUT and D-type can each be configured using a pair of adjacent cells and interconnected by abutment as shown in Fig. 13(b), incurring no additional local routing overhead. As they are approximately the same complexity, one cell-pair and a BLE are assumed to occupy equal area in the same technology.

2) We assumed the impact of the configuration mechanisms to be approximately the same in each case so that it could be ignored. Each PMA block requires 128 configuration bits. Conventional FPGA devices use sparse encodings, typically using at least a factor of 2-4 more configuration bits than necessary, so are likely to exhibit similar a number of configuration bits as the PMA for an equivalent function.

3) The width of the routing channels was estimated to be four times the original logic block width based on data from [44] with the LUT input size (K) within the range 3-6. The overall cost of using a BLE was therefore fixed at 25 units. This is an underestimate for FPGA devices.

4) The routing overhead for the PMA layout was estimated using the stochastic wire length model of [45] with the Rent parameter set to 0.8 . This is an overestimate, more typical of random logic blocks. The true parameter will be smaller for all these circuits. The wire length estimates (in units of cell pitches) were then scaled up by $\sqrt{2}$ to allow for the rectangular routing constraints and doubled again to cover routing congestion and placement inefficiencies. The overall cost of using a polymorphic cell-pair is therefore taken to be $(2 \sqrt{2} \bar{R}+1)$ units, where $\bar{R}$ is the average interconnect length. Routing is confined to the adjacent regions between merged cells as shown in Fig. 10 .

Unlike an FPGA, this array is not constrained by a fixed channel width set by the number of pins on a fixed logic block. The ability to configure routing only where necessary and to collapse low fan-out cells into blocks connected by abutment, results in area ratios less than one for all of the smaller benchmarks. The ratio of the areas reaches (and slightly exceeds) unity 


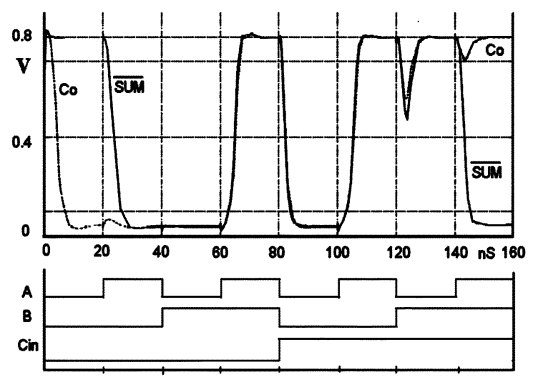

Fig. 12. Simulated sum and carry-out waveforms for full-adder $\left(V_{\mathrm{DD}}=\right.$ $0.8 \mathrm{~V})$.

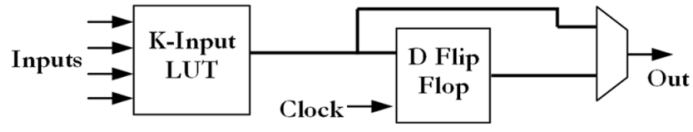

(a)

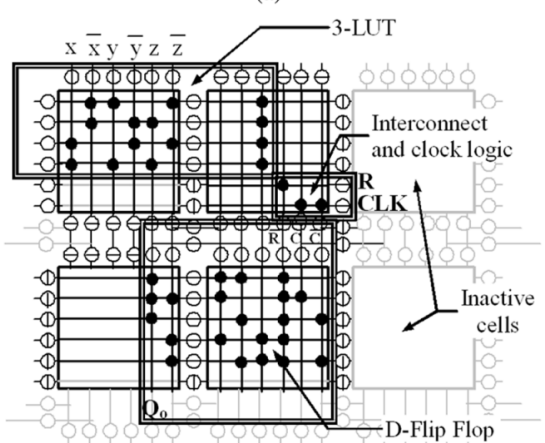

(b)

Fig. 13. (a) BLE structure. (b) Group of configured logic cells forming merged 3-LUT and flip-flop elements. A key difference from (a) is that both the LUT and register outputs are available and it is not necessary to instantiate the multiplexor.

TABLE II

AREA COMPARISON BETWEEN BLE AND THE PMA FOR LGSYNTH93 CIRCUITS [43]

\begin{tabular}{|l||c|c|c||c|c||c|}
\hline \multirow{2}{*}{ Circuit } & \multicolumn{3}{c|}{ Component } & \multicolumn{2}{c||}{ Area } & \\
\cline { 2 - 7 } & $\begin{array}{c}\text { LUT } \\
\text { only }\end{array}$ & $\begin{array}{c}\text { Latch } \\
\text { only }\end{array}$ & $\begin{array}{c}\text { Both } \\
\text { used }\end{array}$ & BLE & PMA & $\frac{\text { Area PMA }}{\text { Area BLE }}$ \\
\hline ex5p.net & 1064 & - & - & 26600 & 15758 & 0.6 \\
apex4.net & 1262 & - & - & 31550 & 19457 & 0.6 \\
misex3.net & 1397 & - & - & 34925 & 22063 & 0.6 \\
alu4.net & 1522 & - & - & 38050 & 24532 & 0.6 \\
des.net & 1591 & - & - & 39775 & 25917 & 0.7 \\
seq.net & 1750 & - & - & 43750 & 29165 & 0.7 \\
apex2.net & 1878 & - & - & 46950 & 31833 & 0.7 \\
spla.net & 3690 & - & - & 92250 & 73757 & 0.8 \\
pdc.net & 4575 & - & - & 114375 & 96463 & 0.8 \\
\hline s298.net & 1923 & 1 & 7 & 48275 & 33067 & 0.7 \\
bigkey.net & 1482 & - & 224 & 42650 & 31969 & 0.7 \\
diffeq.net & 1120 & 3 & 374 & 37425 & 30024 & 0.8 \\
tseng.net & 662 & 1 & 384 & 26175 & 21109 & 0.8 \\
frisc.net & 2670 & 17 & 869 & 88900 & 87540 & 1.0 \\
dma.net & 8350 & 2 & 31 & 209575 & 206658 & 1.0 \\
elliptic.net & 2482 & 2 & 1120 & 90100 & 93863 & 1.0 \\
s38417.net & 4943 & 310 & 1153 & 160150 & 171272 & 1.1 \\
\hline
\end{tabular}

in the larger circuits with longer average wire lengths and in the case where a greater number of flip-flops increases the overall number of cells compared to the FPGA. Even though these results are only approximate, we observe that the array is likely to be no worse that the fixed channel FPGA and will typically be better due to its greater flexibility in trading logic for interconnect area.

The general trend of these results is reinforced in Table III, which shows a similar analysis of a number of arithmetic cir-
TABLE III

Relative AREA FOR ARITHMETIC CIRCUITS

\begin{tabular}{|c|c|c|c|c|c|}
\hline \multirow[t]{2}{*}{ Circuit } & \multirow[b]{2}{*}{ LUTS } & \multirow[b]{2}{*}{ F/Fs } & \multicolumn{2}{|c|}{ Area } & \multirow[b]{2}{*}{$\frac{\text { Area PMA }}{\text { Area BIF }}$} \\
\hline & & & BLE & PMA & \\
\hline 64-bit ripple adder & 165 & 0 & 4125 & 1189 & 0.3 \\
\hline $16 \times 16 \mathrm{add} / \mathrm{shift}$ mult'r & 85 & 51 & 3400 & 861 & 0.3 \\
\hline 32×32 Booth mult'r & 263 & 103 & 9150 & 2903 & 0.3 \\
\hline $32 \times 32$ array mult'r & 2439 & 0 & 60975 & 31857 & 0.4 \\
\hline
\end{tabular}

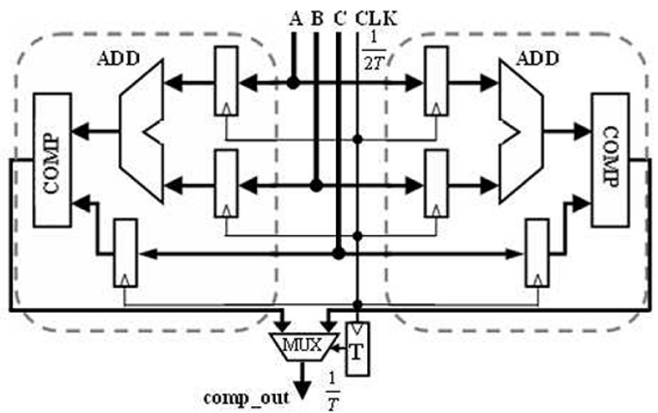

A B C CLK

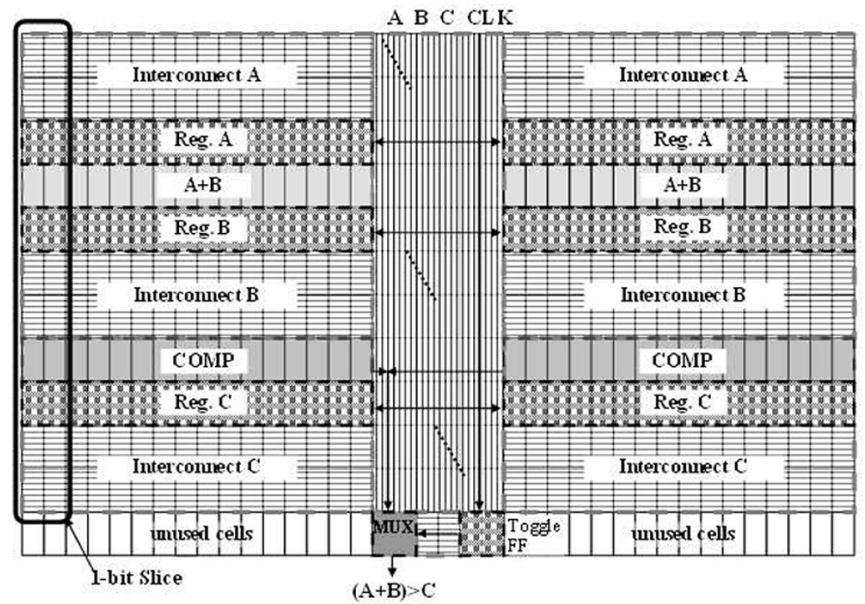

Fig. 14. Example floorplan of a duplicated datapath from [46].

cuits written in VHDL, originally targeting an FPGA. In this case, wire lengths are estimated using a Rent exponent of 0.4 to reflect the regularity of these arithmetic circuits and their low fan-out. These arithmetic circuits can therefore be readily grouped to form larger blocks, mainly by abutment and with a small ratio of internal routing. Table III shows that under the same assumptions as before this regularity and locality will support compact layouts, up to three times more compact than on conventional organizations with fixed routing.

As an example, a duplicated 8-bit data path was set up with the general floorplan shown in Fig. 14. Here, the two data path blocks are formed from replicated 1-bit add/compare/register slices (cf. [46]) mirrored across a common central interconnection bus. Adjacent data path blocks are connected by abutment and interconnect channels are shared where appropriate, thereby incurring little additional interconnection area or delay penalty apart from a small increase in the capacitive load on the "corner" routing cells in the central channel. This takes advantage of a characteristic of the particular organization chosen for this array, i.e., that the dataflow direction can be reversed simply by shifting the slice mapping by one cell position in any direction (see Fig. 9). Of course, the tight rectangular packing exhibited here is a particular characteristic of this example and would not be universal. However, this simple example does serve to il- 
TABLE IV

Simulated Performance Figures for the ARray (WITH FIGURES FROM [41] AND [42] FOR COMPARISON)

\begin{tabular}{|l|c|c||c|c|}
\hline & $\begin{array}{c}\text { D-type } \\
\text { using [25] }\end{array}$ & $\begin{array}{c}\text { Full adder } \\
\text { (Section 3) }\end{array}$ & $\begin{array}{c}\text { Full adder } \\
\text { from [41] }\end{array}$ & $\begin{array}{c}\text { NW Array } \\
\text { from [42] }\end{array}$ \\
\hline Delay & $480 \mathrm{ps}^{\dagger}$ & $5.2 \mathrm{nS}$ & $491 \mathrm{ps}$ & $5-10 \mathrm{nS} \S$ \\
Cycle time & - & - & $1 \mathrm{~ns}$ & - \\
\hline Area & $2.88 \mu m^{2} \ddagger$ & $2.88 \mu m^{2} \ddagger$ & $3 \mu m^{2}$ & $5.6 \mu^{2} \star$ \\
$\begin{array}{l}\text { Drivers/sense } \\
\text { amps }\end{array}$ & - & - & $70 \mu m^{2}$ & - \\
\hline Static Power & $35.9 \mu \mathrm{W}$ & $0.16 \mu \mathrm{W}$ & $0.55 \mu \mathrm{W}$ & $0.1 \mu \mathrm{W}$ \\
Drivers/sense & - & - & $68.72 \mu \mathrm{W}$ & - \\
amps & & & & \\
\hline
\end{tabular}

$\dagger$ - propagation delay: clock $\uparrow$ to $\mathrm{Q}$

\pm - estimate based on $22 \mathrm{~nm}$ design rules with $W_{P, N}=2, L_{P, N}=1$. Each function

occupies two adjacent cells.

$\S$ - static logic case, FO-4. Dynamic logic case is $\sim 10 \mathrm{x}$ faster.

$\star$ - estimate based on $40 \mathrm{OR}$-terms at $22 \mathrm{~nm}$ lithography.

$\diamond-$ value from [6] with $V_{\mathrm{DD}}=1 \mathrm{~V}$.

lustrate how the "polymorphic" behavior of these cells allows a fine-grained organization to efficiently support regular datapath structures.

\section{Static Power-Delay Performance}

In this section, we briefly examine the static power and performance results for the proposed array and compare it against two proposals for nanoscale systems that rely on a partial selfassembly approach. The results are shown in Table IV. The crossbar full-adder circuit of [41] is based on rectifying junctions formed from rotaxane molecules such as those described in [47], whereas the programmable logic array (PLA) circuits in [42] are based on (hypothetical) stochastic self-assembly techniques that would form orthogonal arrays of silicon nanowires randomly connected to a micro-scale address decoder.

Although the nanowire and the molecular crossbar circuits are similar in both functional complexity and in overall size, we have to exercise care when making direct comparisons between them as all of the figures listed depend on specific design details that are difficult to quantify in general terms. The thin-body MOSFETs described in [25] exhibit low values of threshold (particularly the n-type devices) due to the particular gate workfunction chosen. As a result, the static power $\left(I_{\mathrm{OFF}} V_{\mathrm{DD}}\right)$ of the D-type is more than 60 times that of the molecular design and is certainly too high to support the levels of integration envisaged. For example, if we assume that the target is a low-power portable system with a static power target of $0.01 \mathrm{~W} / \mathrm{cm}^{2}$, the density would be power-limited to fewer than 300 LUT blocks $/ \mathrm{cm}^{2}$.

In a fixed threshold system, ultimate scaling is likely to require separate gate work functions set above and below the midband in order to "tune" and balance the transistor thresholds [17]. The silicide devices presented in Section II exhibit much lower values of $I_{\mathrm{OFF}}$ (in the order of $10^{-12} \mathrm{~A}$ ), thus their static power performance is better than even the molecular case, but at the expense of performance $\left(\propto I_{\mathrm{ON}}\right)$. It is worth noting that even at its quoted figure of $0.55 \mu \mathrm{W}$, the molecular crossbar element analyzed in [41] would reach a static power density of $0.01 \mathrm{~W} / \mathrm{cm}^{2}$ at fewer than $2 \times 10^{4} \mathrm{LUT}$ blocks $/ \mathrm{cm}^{2}$, a figure that could be easily exceeded by current low-power CMOS techniques. Once the drivers/sense amplifiers are factored into the molecular crossbar case, its static power is almost double that of the high speed CMOS circuits with approximately the same propagation delay. It is only at the maximum figure for pas- sive cooling of $100 \mathrm{~W} / \mathrm{cm}^{2}$ that we start to approach the sort of densities promised by the device roadmaps [e.g., $O\left(2 \times 10^{8}\right)$ blocks $/ \mathrm{cm}^{2}$ ]. It should be remembered that this not include the effect of dynamic power, which would apply additional constraints. No static power figures are presented in [42] (and the proposed manufacturing methodology is purely speculative in any case). The $0.1 \mu \mathrm{W}$ figure in Table IV is from [6] and is based on the estimated worse-case pull-down resistance for the "nMOS-style" organization and with a supply voltage of $1 \mathrm{~V}$. Nanowire FETs are constrained by the same $I_{\mathrm{ON}}, I_{\mathrm{OFF}}$, and subthreshold slope considerations as conventional devices. As a result, the more complete proposal presented in [42] uses dynamic logic as a way to maintain performance while minimizing overall system power, although at the expense of introducing additional multiphase clock distribution overheads, and with little likely impact on static power.

As a final observation, it is worth noting that by the final ITRS technology nodes (16-22 nm), all of these proposals appear to result in similar device densities and are limited by power/energy rather than layout area. The area comparison presented in Section III-C implies that there is likely to be minimal differences in mapping efficiency when each is applied to real circuits so it will be power density that sets an ultimate scaling limit-supporting perhaps a density factor of $\times 2$ at best. It could be argued that these modest returns would not be worth the significant increase in complexity that would result from mixing conventional and self-assembled manufacturing techniques.

\section{CONCLUSION}

A fine-grained reconfigurable platform based on complementary, DG, fully depleted SOI transistors has been proposed and analyzed. DG-SOI technology is of interest as it offers a way of avoiding short channel effects and substrate leakage as channel lengths are scaled down. Given that the second gate can be justified on these grounds, the ability to configure the transistors' operating region can be added with little additional overhead.

In the fabric developed here, the DG devices are arranged such that the top gates may be programmed to affect the switching threshold of the device and therefore its logic function. Organizing these into simple arrays supports the development of more complex computing functions. While there are still many technical challenges to be overcome, such devices offer a number of tangible benefits, not the least of which is a plausible migration path from conventional (micro-scale) CMOS.

Extending CMOS into the nanoscale domain will require a careful balance between power and performance. The SOI array offers low-power, complementary operation, without the additional overheads of either level restoration or gain blocks (as required, for example, by molecular logic circuits). By comparing the performance of our CMOS array with others based on self-assembly methods, we can see that moving to non-CMOS technologies will not guarantee, per se, higher densities or improved performance. Many of the gains to be made towards the end of the roadmap will be achieved by exploiting innovative architectures to ameliorate the impact of poor device performance and reliability, increasing variability and, in particular, power/energy.

\section{REFERENCES}

[1] S. Hauck, "The roles of FPGAs in reprogrammable systems," Proc. IEEE, vol. 86, no. 4, pp. 615-638, Apr. 1998. 
[2] V. Betz and J. Rose, "How much logic should go in an FPGA logic block?," IEEE Des. Test Comput., vol. 15, no. 1, pp. 10-15, Jan./Mar. 1998.

[3] R. Compano, "Technology roadmap for nanoelectronics," European Commission IST Programme-Future and Emerging Technologies, 2000 [Online]. Available: http://cordis.europa.eu/ist/fet/nidqf.htm

[4] R. Ronen, A. Mendelson, K. Lai, S.-L. Lu, F. Pollack, and J. P. Shen, "Coming challenges in microarchitecture and architecture," Proc. IEEE, vol. 98 , no. 3, pp. 325-340, Mar. 2001

[5] S. C. Goldstein and M. Budiu, "Nanofabrics: Spatial computing using molecular electronics," in Proc. 28th Int. Symp. Comput. Arch., 2001, pp. $178-189$.

[6] A. DeHon, "Array-based architecture for FET-based, nanoscale electronics," IEEE Trans. Nanotechnol., vol. 2, no. 1, pp. 23-32, Mar. 2003.

[7] C. S. Lent, P. D. Tougaw, W. Porod, and G. H. Bernstein, "Quantum cellular automata," Nanotechnol., vol. 4, no. 1, pp. 49-57, 1993.

[8] R. Richter, H. Boeve, L. Bär, J. Bangert, G. Rupp, G. Reiss, and J. Wecker, "Field programmable spin-logic realized with tunnelling-magnetoresistance devices," Solid-State Electron., vol. 46, no. 1-3, pp. 639-643, 2002.

[9] Semiconductor Industry Association, "International Technology Roadmap for Semiconductors," (2005). [Online]. Available: http://public.itrs.net/Common/2005ITRS/Home2005.htm

[10] V. V. Rakitin and E. I. Filippov, "Logical elements based on dual MOS transistors," (1996). [Online]. Available: http://www.niifp.ru/english/ nano/lebdmos.html

[11] A. R. Brown, J. R. Watling, and A. Asenov, "A 3-D atomistic study of archetypal double gate MOSFET structures," J. Computational Electron., vol. 1, no. 1-2, pp. 165-169, 2002.

[12] K. W. Guarini, P. M. Solomon, Y. Zhang, K. K. Chan, E. C. Jones, G. M. Cohen, A. Krasnoperova, M. Ronay, O. Dokumaci, J. J. Bucchignano, J. C. , Jr, C. Lavoie, V. Ku, D. C. Boyd, K. S. Petrarca, I. V. Babich, J. Treichler, and P. M. Kozlowski, "Triple-self-aligned, planar double-gate MOSFETs: Devices and circuits," in Proc. Int. New Electron Devices Meet., 2001, pp. 19.2.1-19.2.4.

[13] J. G. Fossum, M.-H. C. L. Ge, V. P. Trivedi, M. M. Chowdhury, L. Mathew, G. O. Workman, and B.-Y. Nguyen, "A process/physics-based compact model for nonclassical CMOS device and circuit design," Solid-State Electron., vol. 48, no. 6, pp. 919-926, Jun. 2004.

[14] Z. Ren, R. Venugopal, S. Datta, M. Lundstrom, D. Jovanovic, and J. Fossum, "The ballistic nanotransistor: A simulation study," in Proc. Int. Electron Devices Meet., 2000, pp. 715-18.

[15] M. Ieong, E. C. Jones, T. Kanarsky, Z. Ren, O. Dokumaci, R. A. Roy, L. Shi, T. Furukawa, Y. Taur, R. J. Miller, and H.-S. P. Wong, "Experimental evaluation of carrier transport and device design for planar symmetric/asymmetric double-gate/ground-plane CMOSFETs," in Proc. Int. Electron Devices Meet., 2001, pp. 6.1-6.4.

[16] N. J. Collier and J. R. A. Cleaver, "Novel dual-gate HEMT utilising multiple split gates," Microelectron. Eng., vol. 41-42, pp. 457-460, 1998.

[17] L. Chang, S. Tang, T.-J. King, J. Bokor, and C. Hu, "Gate length scaling and threshold voltage control of double-gate MOSFETs," in Proc. Int. Electron Devices Meet., 2000, pp. 719-722.

[18] T. Schulz, W. Rosner, E. Landgraf, L. Risch, and U. Langmann, "Planar and vertical double gate concepts," Solid-State Electron., vol. 46, no. 7, pp. 985-989, 2002.

[19] F. Allibert, A. Zaslavsky, J. Pretet, and S. Cristoloveanu, "Double-gate MOSFETs: Is gate alignment mandatory?," in Proc. Euro. Solid-State Device Res. Conf., 2001, pp. 267-270.

[20] J. R. Tucker, "Schottky barrier MOSFETs for silicon nanoelectronics," in Proc. Adv. Workshop Frontiers Electron. (WOFE), 1997, pp. 97-100.

[21] T. Lepselter and S. M. Sze, "SB-IGFET: An insulated-gate field-effect transistor using Schottky barrier contacts for source and drain," Proc. IEEE, vol. 56, no. 8, pp. 1400-1401, Aug. 1968.

[22] Z. Ren, R. Venugopal, S. Datta, and M. Lundstrom, "The ballistic nanotransistor: A simulation study," in Proc. Int. Electron Devices Meeting, 2002, pp. 715-718.

[23] W. Chen, L. F. Register, and S. K. Banerjee, "Simulation of quantum and scattering effects along the channel of ultra-scaled si-based MOSFETs," in Proc. 60th Device Res. Conf. Dig., 2002, pp. 109-110.

[24] Silvaco, Santa Clara, CA, "Atlas device simulation software," 2007 [Online]. Available: http://www.silvaco.com

[25] J. Kedzierski, P. Xuan, E. H. Anderson, J. Bokor, T.-J. King, and C. $\mathrm{Hu}$ " "Complementary silicide source/drain thin-body MOSFETs for the 20 nm gate length regime," in Proc. Int. Electron Dev. Meet. (IEDM), 2000, pp. 57-60.

[26] Q.-T. Zhao, P. Kluth, S. Winnerl, and S. Mantl, "Fabrication of Schottky barrier MOSFETs on SOI by a self-assembly CoSi2-patterning method," Solid-State Electron., vol. 2003, pp. 1183-1186.

[27] S. Zhu, J. Chen, M.-F. Li, S. J. Lee, J. Singh, C. X. Zhu, A. Du, C. H. Tung, A. Chin, and D. L. Kwong, "N-type Schottky barrier source/drain MOSFET using ytterbium silicide," IEEE Electron Device Lett., vol. 25 , no. 8 , pp. 565-567, Aug. 2004.
[28] H.-S. P. Wong, D. J. Frank, and P. M. Solomon, "Device design considerations for double-gate, ground-plane, and single-gated ultra-thin SOI MOSFET's at the $25 \mathrm{~nm}$ channel length generation," in Proc. Int. Electron Devices Meet. (IEDM), 1998, pp. 407-410.

[29] J. R. Watling, A. R. Brown, and A. Asenov, "Can the density gradient approach describe the source-drain tunnelling in decanano double-gate MOSFETs?," J. Comput. Electron., vol. 1, pp. 289-293, 2002.

[30] D. J. Wouters, J.-P. Colinge, and H. E. Maes, "Subthreshold slope in thin-film SOI MOSFETs," IEEE Trans. Electron Devices, vol. 37, no. 9, pp. 2022-2033, Sep. 1990

[31] J. Kedzierski, E. Nowak, T. Kanarsky, Y. Zhang, D. Boyd, R. Carruthers, C. Cabral, R. Amos, C. Lavoie, R. Roy, J. Newbury, E. Sullivan, J. Benedict, P. Saunders, K. Wong, D. Canaperi, M. Krishnan, K.-L. Lee, B. A. Rainey, D. Fried, P. Cottrell, H.-S. P. Wong, M. Ieong, and W. Haensch, "Metal-gate finfet and fully-depleted SOI devices using total gate silicidation," in Proc. Int. Electron Devices Meet. (IEDM), 2002, pp. 247-250.

[32] Q. Chen, L. Wang, and J. D. Meindl, "Impact of high-k dielectrics on undoped double-gate MOSFET scaling," in Proc. IEEE Int. SOI Conf., 2002, pp. 115-116.

[33] X. Lin, M. Chan, and H. Wang, "Opposite side floating gate SOI flash memory cell," in Proc. IEEE Electron Devices Meet., 2000, pp. 12-15.

[34] P. Beckett, "A fine-grained reconfigurable logic array based on double gate transistors," in Proc. IEEE Int. Conf. Field-Program. Technol. (FPT), 2002, pp. 260-267.

[35] P. Beckett, "A polymorphic hardware platform," in Proc. 10th Reconfigurable Arch. Workshop (RAW), 2003, p. 175.

[36] K. Chen, C. Hu, P. Fang, M. R. Lin, and D. L. Wollesen, "Predicting CMOS speed with gate oxide and voltage scaling and interconnect loading effects," IEEE Trans. Electron Devices, vol. 44, no. 11, pp. 1951-1957, Nov. 1997

[37] T. Sakurai and A. Newton, "Alpha-power law MOSFET model and its applications to CMOS inverter delay and other formulas," IEEE $J$. Solid-State Circuits, vol. 25, no. 2, pp. 584-594, Feb. 1990.

[38] S.-M. Kang and Y. Leblebici, CMOS Digital Integrated Circuits: Analysis and Design. New York: McGraw-Hill, 1996.

[39] D. J. Frank, R. H. Dennard, E. Nowak, P. M. Solomon, Y. Taur, and H.-S. P. Wong, "Device scaling limits of si MOSFETs and their application dependencies," Proc. IEEE, vol. 89, no. 3, pp. 259-288, Mar. 2001.

[40] "The nanotechnology simulation hub," [Online]. Available: http://www.nanohub.org/

[41] M. M. Ziegler and M. R. Stan, "The CMOS/nano interface from a circuits perspective," in Proc. Int. Symp. Circuits Syst. (ISCAS), 2003, pp. 904-907.

[42] A. DeHon and M. J. Wilson, "Nanowire-based sublithographic programmable logic arrays," in Proc. ACM/SIGDA 12th Int. Symp. FieldProgram. Gate Arrays (FPGA), 2004, pp. 123-132.

[43] "The FPGA place-and-route challenge," [Online]. Available http://www.eecg.toronto.edu/ vaughn/challenge/challenge.html

[44] J. Rose, R. J. Francis, D. Lewis, and P. Chow, "Architectures of fieldprogrammable gate arrays: The effect of logic functionality on area efficiency," IEEE J. Solid-State Circuits, vol. 25, no. 5, pp. 1217-1225, May 1990.

[45] J. A. Davis, V. K. De, and J. D. Meindl, "A stochastic wire-length distribution for gigascale integration (gsi). ii. applications to clock frequency, power dissipation, and chip size estimation," IEEE Trans. Electron Devices, vol. 45, no. 3, pp. 590-597, Mar. 1998.

[46] A. P. Chandrakasan, S. Sheng, and R. W. Brodersen, "Low-power CMOS digital design," IEEE J. Solid-State Circuits, vol. 27, no. 4, pp. 473-484, Apr. 1992.

[47] C. P. Collier, E. W. Wong, M. Belohradský, F. M. Raymo, J. F. Stoddart, P. J. Kuekes, R. S. Williams, and J. R. Heath, "Electronically configurable molecular-based logic gates," Science, vol. 285, pp. 391-394, 1999.

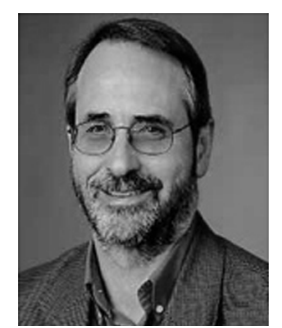

Paul Beckett (M'06) was born in Melbourne, Australia, in 1953. He received the B.Eng. (Comm.), M.Eng., and Ph.D. degrees from the Royal Melbourne Institute of Technology (now RMIT University), Melbourne, in 1975, 1984, and 2007, respectively.

He is currently a Senior Lecturer with the School of Electrical and Computer Engineering, RMIT University, where he teaches undergraduate and postgraduate courses in embedded computer architecture, digital logic, and VLSI design. His research interests include the design and simulation of nanoscale devices and the mixed-signal modeling of reconfigurable circuits and architectures. 